\title{
Measurement of the hadronic cross section at KLOE/KLOE-2
}

\author{
Veronica De Leo ${ }^{1, a}$ on behalf of the KLOE/KLOE-2 Collaborations \\ ${ }^{1}$ INFN Sezione Roma Tre, Via della Vasca Navale 84, 00146 Roma, Italy
}

\begin{abstract}
The measurement of the hadronic cross section $\sigma\left(e^{+} e^{-} \rightarrow \pi^{+} \pi^{-}\right)$is particularly important to test the Standard Model calculation for the $(g-2)$ of the muon, where a long standing $3 \sigma$ discrepancy is observed. Different measurements of this quantity have been performed at $D A \Phi N E$, the Frascati $\phi$ factory, with the KLOE detector. In 2005, 2008, 2013 the KLOE/KLOE-2 Collaborations published three measurements of the $\sigma\left(e^{+} e^{-} \rightarrow \pi^{+} \pi^{-}\right)$cross section with the photon emitted at small angle, and an independent measurement of the $\sigma\left(e^{+} e^{-} \rightarrow \pi^{+} \pi^{-}\right)$cross section with the photon emitted at large angle using data at a collision energy of 1 $\mathrm{GeV}$ (i.e. $20 \mathrm{MeV}$ below the $\phi$-peak) was published in 2010. In the following the preliminary combination of the last three KLOE results (KLOE08, KLOE10, KLOE12), together with the preliminary fit to the pion form factor, will be presented.
\end{abstract}

\section{Introduction}

The anomalous magnetic moment of the muon, $a_{\mu}$, is one of the best known quantities in particle physics. The comparison of the theoretical and measured values of $a_{\mu}$ shows a discrepancy of about $3 \sigma$ [1]. The Standard Model (SM) prediction for $a_{\mu}^{S M}$ is generally divided into three parts:

$$
a_{\mu}^{S M}=a_{\mu}^{Q E D}+a_{\mu}^{E W}+a_{\mu}^{H a d}
$$

The QED part includes all photonic and leptonic (e, $\mu$, $\tau)$ loops starting with the classic $\alpha / 2 \pi$ Schwinger contribution. Loop contributions involving heavy $W^{ \pm}, Z$ or Higgs particles are collectively labeled as $a_{\mu}^{E W}$. The hadronic contribution itself is given by different contributions:

$$
a_{\mu}^{\mathrm{Had}}=a_{\mu}^{\mathrm{Had}, \mathrm{LO}}+a_{\mu}^{\mathrm{Had}, \mathrm{HO}}+a_{\mu}^{\mathrm{Had}, \mathrm{LBL}}
$$

where $a_{\mu}^{H a d, L O}$ is the lowest-order contribution from hadronic vacuum polarization, $a_{\mu}^{\mathrm{Had}, \mathrm{HO}}$ is the corresponding higher-order part and the last term $a_{\mu}^{H a d, L B L}$ is the lightby-light (LBL) scattering part [2]. A large part of the uncertainty on the theoretical estimates comes from the lead-

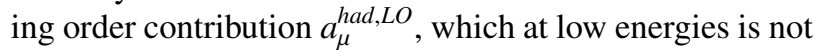
calculable by perturbative QCD, but has to be evaluated with a dispersion integral using measured hadronic cross sections:

$$
a_{\mu}^{H a d}[L O]=\frac{1}{3}\left(\frac{a}{\pi}\right)^{2} \int_{m_{\pi}^{2}}^{\infty} d s \frac{K(s)}{s} R(s) .
$$

$K(s)$ is a QED kernel function [3] and $R(s)$ denotes the ratio of the cross section for $e^{+} e^{-}$annihilation into hadrons to the pointlike muon-pair cross section at center of mass

\footnotetext{
a e-mail: veronica.deleo@ roma3.infn.it
}

energy $\sqrt{s}$. The integration kernels occurring in the dispersion relations emphasise low photon virtualities, due to the $1 / \mathrm{s}$ slope of the cross section.

Therefore, improved precision in the $\pi \pi$ cross section would result in a reduction of the uncertainty on the LO hadronic contribution to $a_{\mu}$, and in turn to the SM prediction for $a_{\mu}$. The measurement of the $\sigma\left(e^{+} e^{-} \rightarrow \pi^{+} \pi^{-}\right)$ cross section allows to determine the pion form factor $\left|F_{\pi}\right|^{2}$ and the two pion contribution to the muon anomaly $a_{\mu}$. Such a measurement has been performed with the KLOE detector at $D A \Phi N E$, the Frascati $\phi$-factory.

\section{Measurement of $\sigma_{\pi \pi}$ with ISR}

The KLOE experiment is the first to have employed Initial State Radiation (ISR) to precisely determine the $e^{+} e^{-} \rightarrow$ $\pi^{+} \pi^{-}(\gamma)$ cross section below $1 \mathrm{GeV}$. The dipion cross section has been obtained from the following relation:

$$
\left.s \frac{d \sigma\left(\pi^{+} \pi^{-} \gamma\right)}{d \sigma_{\pi}}\right|_{I S R}=\sigma_{\pi \pi}\left(s_{\pi}\right) H\left(s_{\pi}, s\right),
$$

where the radiator function $H$ is computed from QED with complete NLO corrections and depends on the $e^{+} e^{-}$center of mass energy squared $s$. $\sigma_{\pi \pi}$ obtained from Eq. (4) requires accounting for final state radiation (FSR). An alternative way to extract the $\pi \pi$ cross section uses the $\pi^{+} \pi^{-} \gamma / \mu^{+} \mu^{-} \gamma$ ratio. Eq. (4) is also valid for $e^{+} e^{-} \rightarrow$ $\mu^{+} \mu^{-} \gamma$ and $e^{+} e^{-} \rightarrow \mu^{+} \mu^{-}$with the same radiator function $H$.

The pion form factor is calculated by the following equation:

$$
\left|F_{\pi}\left(s^{\prime}\right)\right|^{2}=\frac{3}{\pi} \frac{s^{\prime}}{\alpha^{2} \beta_{\pi}^{3}} \sigma_{\pi \pi(\gamma)}^{0}\left(s^{\prime}\right)\left(1+\delta_{V P}\right)\left(1-\eta_{\pi}\left(s^{\prime}\right)\right)
$$


where $\delta_{V P}$ is the Vacuum Polarization (VP) correction, $\eta_{\pi}$ accounts for FSR radiation assuming point-like pions and $\sigma_{\pi \pi}^{0}$ is the bare cross section defined as [16]

$$
\begin{aligned}
& \sigma^{0}\left(\pi^{+} \pi^{-}, s^{\prime}\right)= \\
& \frac{d \sigma\left(\pi^{+} \pi^{-} \gamma, I S R\right) / d s^{\prime}}{d \sigma\left(\mu^{+} \mu^{-} \gamma, I S R\right) / d s^{\prime}} \times \sigma^{0}\left(e^{+} e^{-} \rightarrow \mu^{+} \mu^{-} \gamma, s^{\prime}\right)
\end{aligned}
$$

where $s^{\prime}=s_{\pi}=s_{\mu}$.

\section{The KLOE detector}

The KLOE detector operates at $D A \Phi N E$, the Frascati $\phi$ factory, an $e^{+} e^{-}$collider running at fixed energy, $W=$ $\sqrt{s} \sim 1020 \mathrm{MeV}$, the $\phi$ meson mass. It consists of a cylindrical drift chamber (DC) [13] and an electromagnetic calorimeter (EMC) [14]. The DC has a momentum resolution of $\sigma_{p_{\perp}} / p_{\perp} \sim 0.4 \%$ for tracks with polar angle $\theta>45^{\circ}$. Track points are measured in the DC with a resolution in $r-\phi$ of $\sim 0.15 \mathrm{~mm}$ and $\sim 2 \mathrm{~mm}$ in $\mathrm{z}$. The EMC has an energy resolution of $\sigma_{E} / E \sim 5.7 \% / \sqrt{E}(\mathrm{GeV})$ and an excellent time resolution of $\sigma_{t} \sim 54 \mathrm{ps} / \sqrt{E}(\mathrm{GeV}) \oplus 100 \mathrm{ps}$. Calorimeter clusters are reconstructed grouping together energy deposits close in space and time. A superconducting coil provides an axial magnetic field of $0.52 \mathrm{~T}$ along the bisector of the colliding beam directions. The bisector is taken as the $\mathrm{z}$ axis of our coordinate system. The $\mathrm{x}$ axis is horizontal, pointing to the center of the collider rings and the $y$ axis is vertical, directed upwards. A cross section of the detector in the $\mathrm{y}, \mathrm{z}$ plane is shown in Fig. 1.

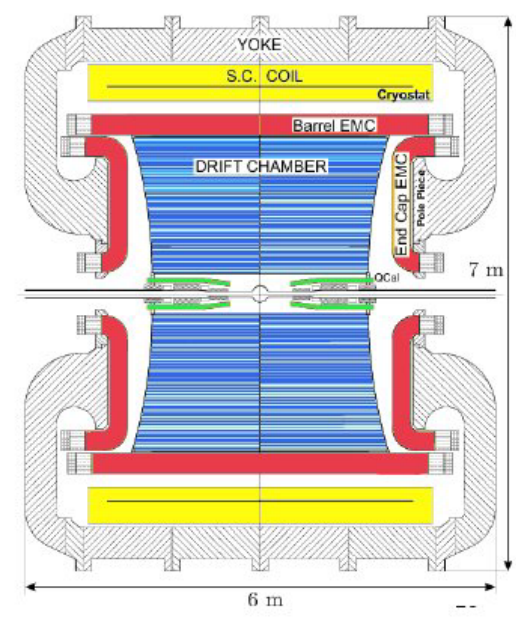

Figure 1. Schematic view of the KLOE detector. In the picture the superconducting coil (yellow), the electromagnetic calorimeter (red) and the cylindrical drift chamber (blue) are visible.

\section{Measurement of the $e^{+} e^{-} \rightarrow \pi^{+} \pi^{-}$cross section at KLOE}

The KLOE08 [15] and KLOE12 [16] analyses used the small angle photon selection. In this case the photon is emitted within a cone of $\theta_{\gamma}<15^{\circ}$ around the beamline (narrow cones in Fig. 2) and the two charged pion tracks have $50^{\circ}<\theta_{\pi}<130^{\circ}$ (wide cones in Fig. 2). This choice for the photon selection implies that it is not explicitly detected so its momentum is reconstructed from kinematics: $\overrightarrow{p_{\gamma}} \simeq \overrightarrow{p_{\text {miss }}}=-\left(\overrightarrow{p_{+}}+\overrightarrow{p_{-}}\right)$. Many advantages derive from the use of these selection cuts as the high statistics for ISR signal events, a reduced contamination from the resonant process $e^{+} e^{-} \rightarrow \phi \rightarrow \pi^{+} \pi^{-} \pi^{0}$ and from the final state radiation process $e^{+} e^{-} \rightarrow \pi^{+} \pi^{-} \gamma_{F S R}$. On the other hand this selection results in a kinematical suppression of events with $M_{\pi \pi}<0.35 \mathrm{GeV}^{2}$, since a highly energetic photon emitted at small angle forces the pions also to be at small angles (and thus outside the selection cuts).

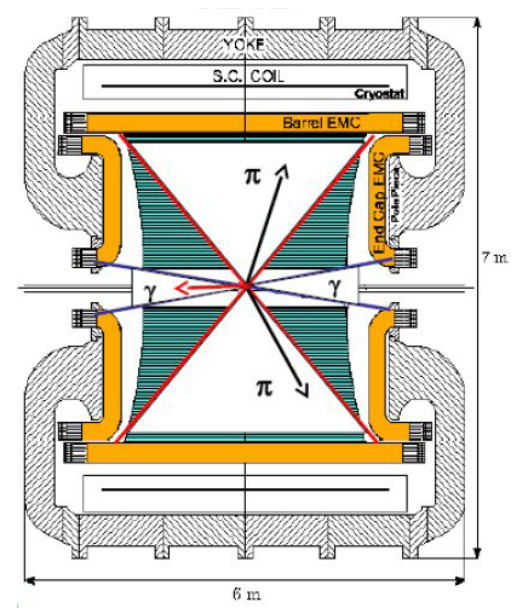

Figure 2. Schematic view of the KLOE detector with selection regions.

The KLOE10 analysis [17] instead was performed requiring events that are selected to have a photon at large polar angles between $50^{\circ}<\theta_{\gamma}<130^{\circ}$ (wide cones in Fig. 2), in the same angular region as the pions.

This selection cut allowed to access the two pion threshold. However, compared to the measurement with photons at small angles, this condition implied a reduction in statistics and an increase of the background from the process $\phi \rightarrow \pi^{+} \pi^{-} \pi^{0}$.

In Fig. 3 the comparison between the measurements of the pion form factor of the KLOE08 and KLOE10 analyses is reported. As one can see, an excellent agreement is found for $\left(M_{\pi \pi}^{0}\right)^{2}>0.5 \mathrm{GeV}^{2}$, while below the KLOE10 result is lower by few percent. We stress that the data sets have been obtained at different operating conditions of the $D A \Phi N E$ collider, and different selection cuts in acceptance were used. Also the analysis procedures were different since in the KLOE08 analysis the radiated photon was not detected.

In Fig. 4, the KLOE10 result is compared with the results obtained from the energy scan experiments CMD-2 $[18,19]$ and SND [20] in Novosibirsk and the result from the BaBar experiment at SLAC [21], which uses the ISR method. Whenever several data points fall in one KLOE bin of $0.01 \mathrm{GeV}^{2}$, the values are statistically averaged. 

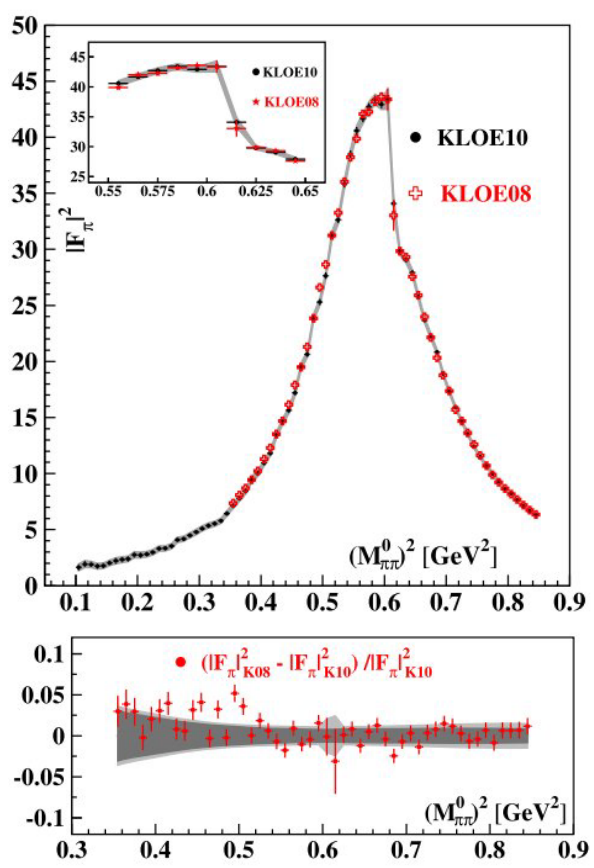

Figure 3. Comparison of KLOE10 result with the previous KLOE08 measurement [15]. Top: pion form factor $\left|F_{\pi}\right|^{2}$. Bottom: fractional difference between KLOE08 and KLOE10 results. The dark (light) gray band gives the statistical (total) error for the present result. Errors on KLOE08 points contain the combined statistical and systematic uncertainty.

The agreement with the SND result is rather good around the $\rho$-peak and at higher energies, while the result from the CMD-2 collaboration is slightly higher than the KLOE10 measurement, confirming the observation already reported in the previous KLOE publication [15]. Below the $\rho$-peak, all three experiments are in agreement within uncertainties. KLOE10 and BaBar results are in agreement within errors below $0.65 \mathrm{GeV}$, while above the new $\mathrm{BaBar}$ measurement is about $2-3 \%$ higher.

\subsection{Measurement of the pion form factor from the $\pi \pi \gamma / \mu \mu \gamma$ ratio}

The data sample corresponds to an integrated luminosity of $239.2 \mathrm{pb}^{-1}$ collected in 2002 (the same used in the KLOE08 analysis).

However, while the analysis for $\pi \pi \gamma$ is essentially the same as for KLOE08, there are some new elements in the $\mu \mu \gamma$ analysis. First of all the separation between the $\pi \pi \gamma$ and $\mu \mu \gamma$ events is obtained by using the track mass variable $\left(M_{T R K}\right)$ with the following conditions: $M_{T R K}<115 \mathrm{MeV}$ for the muons and $M_{T R K}>130$ $\mathrm{MeV}$ for the pions. It is calculated from the energy and momentum conservation laws:

$$
\begin{aligned}
& \left(\sqrt{s}-\sqrt{\left|\overrightarrow{p_{+}}\right|^{2}+M_{T R K}^{2}}-\sqrt{\left|\overrightarrow{p_{-}}\right|^{2}+M_{T R K}^{2}}\right)^{2}- \\
& +\left(\overrightarrow{p_{+}}+\overrightarrow{p_{-}}\right)^{2}=0
\end{aligned}
$$
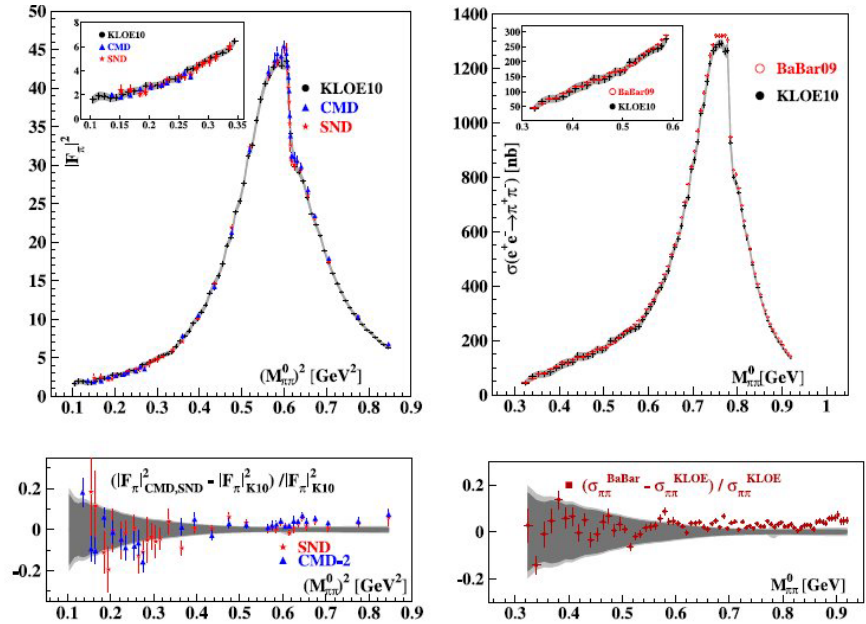

Figure 4. Top left: $\left|F_{\pi}\right|^{2}$ from CMD-2 [18, 19], SND [20] and KLOE10 result as function of $\left(M_{\pi \pi}^{0}\right)^{2}$. Bottom left: fractional difference between CMD-2 or SND and KLOE. Top right: $\sigma_{\pi \pi}^{\text {bare }}$ from BaBar [21] and the KLOE10 result as function of $M_{\pi \pi}^{0}$. Bottom right: fractional difference between BaBar and KLOE. CMD-2, SND and BaBar data points have the total uncertainty attached. The dark (light) band in the lower plots shows statistical (total) error of the KLOE result [17].

where $\overrightarrow{p_{ \pm}}$is the measured momentum of the positive (negative) particle.

This selection has been cross checked using a kinematic fit or applying a quality cut on the helix fit for both $\pi-\mu$ tracks. Consistent results have been obtained with all methods.

The differential $\mu \mu \gamma$ cross section is obtained from the observed number of events $N_{o b s}$, after subtracting the residual background $N_{b k g}$ and dividing for the selection efficiency $\left(\epsilon\left(s_{\mu}\right)\right)$ and luminosity $(L)$, as:

$$
\frac{d \sigma_{\mu \mu \gamma}}{d s_{\mu}}=\frac{N_{o b s}-N_{b k g}}{\Delta s_{\mu}} \frac{1}{\epsilon\left(s_{\mu}\right) L}
$$

The result on the measured $\mu \mu \gamma$ cross section is shown in the top part of Fig. 5, compared with the QED calculations to NLO made by the MC code Phokhara [7].

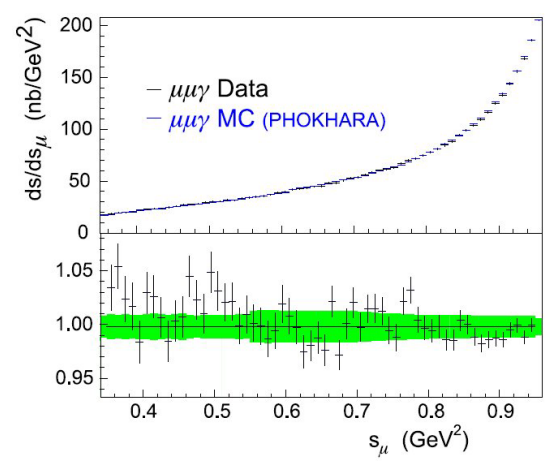

Figure 5. Top: comparison of data and MC results for $d \sigma_{\mu \mu \gamma} / d s_{\mu}$. Bottom: ratio of the two spectra. The green band shows the systematic error [16]. 
Fig. 5, bottom, refers to the ratio between the two differential cross sections. The green band shows the systematic uncertainty, experimental and theoretical, of the measured cross section. The average ratio, using only statistical errors, is $0.9981 \pm 0.0015$, in agreement with the quoted systematic uncertainties [16].

The bare cross section $\sigma_{\pi \pi(\gamma)}^{0}$ (inclusive of FSR, with VP effects removed) is obtained from the bin-by-bin ratio of the $\pi \pi \gamma$ and $\mu \mu \gamma$ differential cross sections described above. This cross section is used in the dispersion integral to compute $\Delta^{\pi \pi} a_{\mu}$. The pion form factor $\left|F_{\pi}\right|^{2}$ is then calculated using Eq. (5).

The dispersion integral for $\Delta^{\pi \pi} a_{\mu}$ is computed as the sum of the values for $\sigma_{\pi \pi(\gamma)}^{0}$ times the kernel $\mathrm{K}(\mathrm{s})$, times $\Delta s=$ $0.01 \mathrm{GeV}^{2}$ :

$$
\Delta^{\pi \pi} a_{\mu}=\frac{1}{4 \pi^{3}} \int_{s_{\min }}^{s_{\max }} d s \sigma_{\pi \pi(\gamma)}^{0}(s) K(s)
$$

where the kernel is given in Ref. [23]. Eq. (9) gives $\Delta^{\pi \pi} a_{\mu}=\left(385.1 \pm 1.1_{\text {stat }} \pm 2.6_{\text {exp }} \pm 0.8_{t h}\right) \times 10^{-10}$ in the interval $0.35<M_{\pi \pi}^{2}<0.95 \mathrm{GeV}^{2}$. For each bin contributing to the integral, statistical errors are combined in quadrature and systematic errors are added linearly.

The comparison between the last $\Delta^{\pi \pi} a_{\mu}$ KLOE measurement and the previous KLOE08 and KLOE10 measurements was made and the results have been reported in the Table 1.

Table 1. Comparison of $\Delta^{\pi \pi} a_{\mu}$ between the KLOE12 and the previous KLOE measurements.

\begin{tabular}{ll}
\hline Measurement & $\Delta a_{\mu}^{\pi \pi}\left(0.35-0.95 \mathrm{GeV}^{2}\right) \times 10^{10}$ \\
\hline KLOE12 & $385.1 \pm 1.1_{\text {stat }} \pm 2.7_{\text {sys }+ \text { theo }}$ \\
KLOE08 & $387.2 \pm 0.5_{\text {stat }} \pm 3.3_{\text {sys }+ \text { theo }}$ \\
\hline & $\Delta a_{\mu}^{\pi \pi}\left(0.35-0.85 \mathrm{GeV}^{2}\right) \times 10^{10}$ \\
\hline KLOE12 & $377.4 \pm 1.1_{\text {stat }} \pm 2.7_{\text {sys }+ \text { theo }}$ \\
KLOE10 & $376.6 \pm 0.9_{\text {stat }} \pm 3.3_{\text {sys }+ \text { theo }}$
\end{tabular}

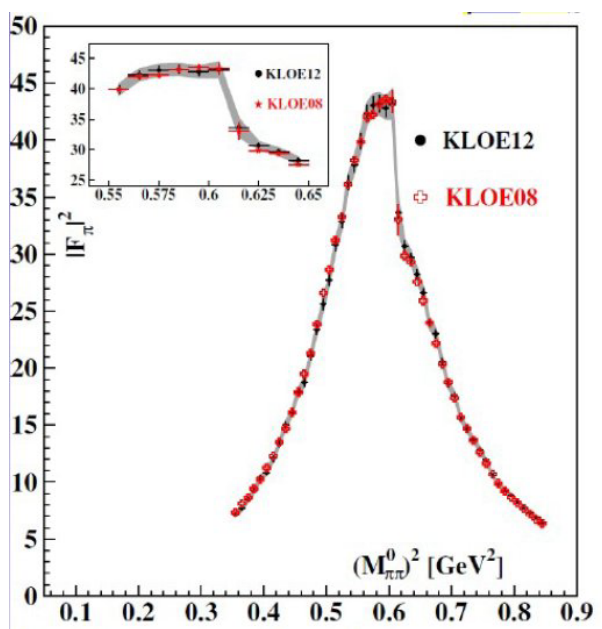

Figure 6. The pion form factor obtained in the KLOE12 (points) and KLOE08 [15] (crosses) measurements.

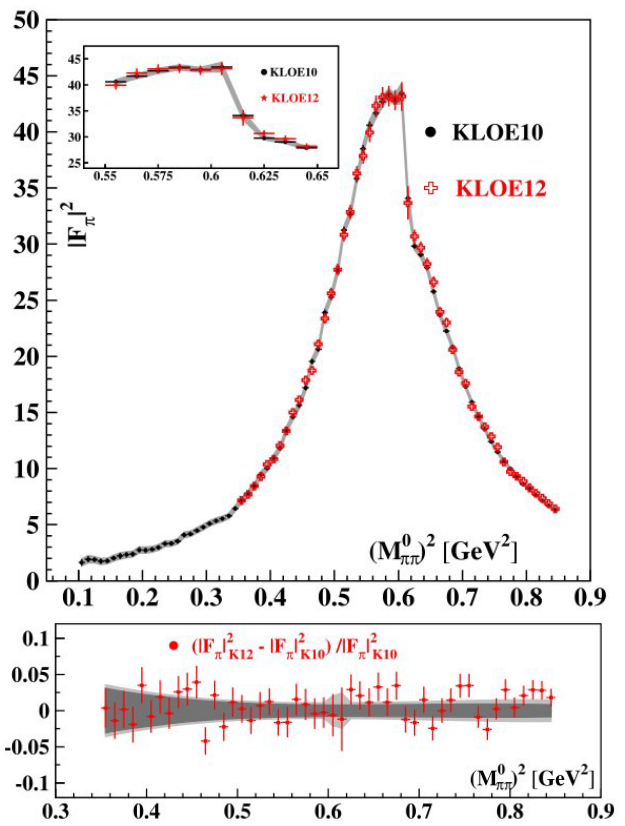

Figure 7. Top: the pion form factor obtained in the KLOE12 work (crosses) and from the measurement with the photon at large angle, KLOE10 [17] (points). Bottom: fractional difference between the two $\left|F_{\pi}\right|^{2}$ measurements. The dark gray band is the statistical error from [17], the light gray band is the combined statistical and systematic uncertainty. In both figures, errors on crosses include combined statistical and systematic uncertainties.

Comparing the results of the KLOE12 and KLOE08 analyses it is possible to see that they are in good agreement, in particular in the $\rho$ mass region (see Fig. 6). An improvement of the systematic error has been reached in the KLOE12 measurement. A sizeable improvement of the theoretical error is achieved thanks to the ratio method. Fig. 7, top, shows the comparison between the $\left|F_{\pi}\right|^{2}$ distribution obtained in the KLOE12 and KLOE10 measurements, requiring the ISR photon to be reconstructed at large angle, inside the EMC barrel. Fig. 7, bottom, shows the fractional difference between the two measurements. They are obtained from independent data sets with different running conditions $\left(\mathrm{W}=M_{\phi} \mathrm{KLOE} 12\right.$, $\mathrm{W}=1 \mathrm{GeV}$ KLOE10), and also with a different selection, that imply independent systematic uncertainties. The two measurements are in very good agreement.

The preliminary combination of the last three KLOE results (KLOE08, KLOE10, KLOE12) [24] is obtained using the Best Linear Unbiased Estimate (BLUE) method $[25,26]$. In Fig. 8, top, the pion form factor measurements for the three KLOE analysis and the fractional difference, bottom, are shown. The following $a_{\pi \pi}^{\mu}$ values are found:

$$
\begin{aligned}
& a_{\pi \pi}^{\mu}\left(0.1-0.95 \mathrm{GeV}^{2}\right)=(487.8 \pm 5.7) \cdot 10^{-10} \\
& a_{\pi \pi}^{\mu}\left(0.1-0.85 \mathrm{GeV}^{2}\right)=(378.1 \pm 2.8) \cdot 10^{-10}
\end{aligned}
$$

The combined measurement of the $\left|F_{\pi}\right|^{2}$ has also been fitted using the Gounaris-Sakurai (GS) model [27] (see Fig. 9). In the table of Fig. 9 preliminary fit results are reported. The determination of the $\omega$-meson mass parame- 


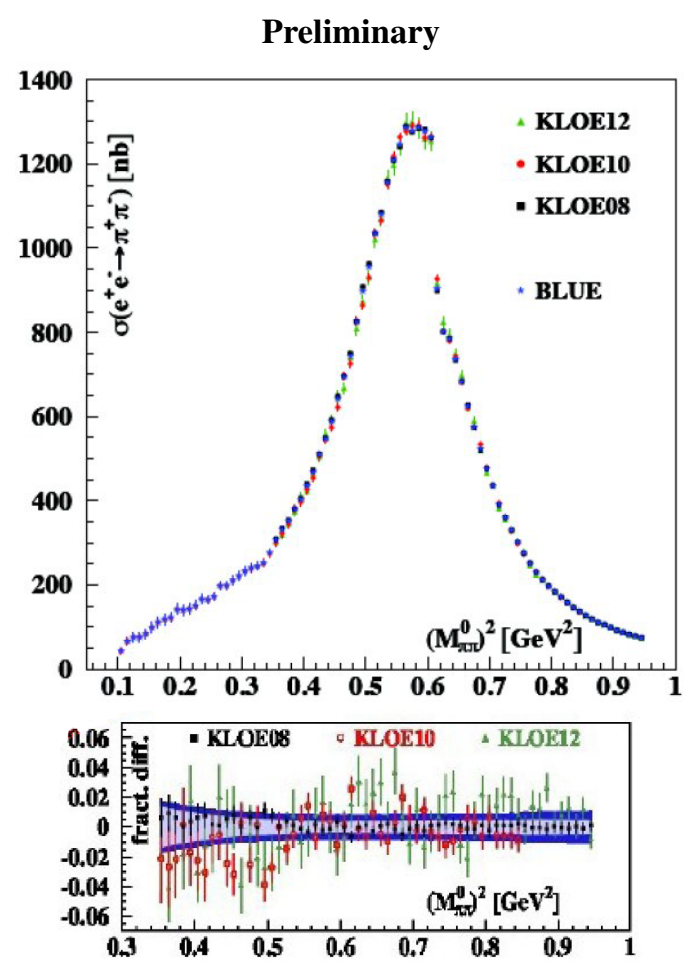

Figure 8. Preliminary combination of the last three KLOE results (KLOE08, KLOE10, KLOE12) on the pion form factor (top) and the fractional difference (bottom) [24] using the Best Linear Unbiased Estimate (BLUE) method [25, 26].

ter is very close to the current PDG value $\left(\mathrm{M}_{\omega}^{K L O E}=782.7\right.$ \pm 0.2stat; PDG $\mathrm{M}_{\omega}^{P D G}=782.65 \pm 0.12$ ) demonstrating the accuracy of the track momentum reconstruction of the KLOE detector.

\section{Conclusions}

During the last 10 years KLOE has performed a series of precision measurements via the Initial State Radiation (ISR). The preliminary combined measurement of the last analysis (KLOE12) with two previously published results (KLOE08, KLOE10), with the corresponding fit using the GS parametrization, has been presented. The result confirms the current discrepancy $(\approx 3 \sigma)$ ) between the Standard Model (SM) calculation and the experimental value of the muon anomaly $a_{\mu}$.

\section{References}

[1] F. Jegerlehner, A. Nyffeler, Phys. Rept. 477 (2009) (Erratum).

[2] M. Davier and W.J. Marciano, Ann. Rev. Nucl. and Part. Sci. 54, 115 (2004).

[3] S.J. Brodsky and E. de Rafael, Phys. Rev. 168, 1620 (1968).

[4] G. Rodrigo, H. Czyż , J.H. Kühn, M. Szopa, Eur. Phys. J. C 24 (2002) 71.

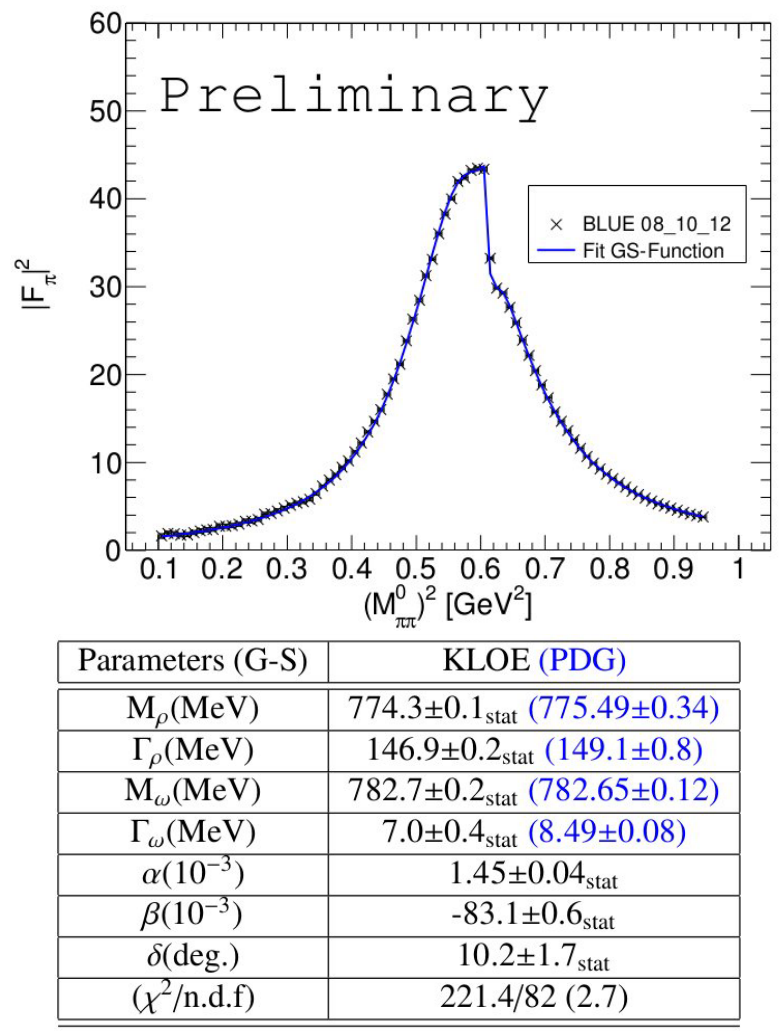

Figure 9. Preliminary fit to the combination of the last three KLOE results (KLOE08, KLOE10, KLOE12) on the $\left|F_{\pi}\right|^{2}$. In the table below the parameters obtained from the fit are reported.

[5] H. Czyż, A. Grzelinska, J.H. Kühn, G. Rodrigo, Eur. Phys. J. C 27 (2003) 563.

[6] H. Czyż, A. Grzelinska, J.H. Kühn, G. Rodrigo, Eur. Phys. J. C 33 (2004) 333.

[7] H. Czyż, A. Grzelinska, J.H. Kühn, G. Rodrigo, Eur. Phys. J. C 39 (2005) 411.

[8] S. Actis, et al., Working Group on Radiative Corrections and Monte Carlo Generators for Low Energies Collaboration, Eur. Phys. J. C 66 (2010) 585.

[9] B. Aubert, et al., BaBar Collaboration, Phys. Rev. Lett.103 (2009) 231801.

[10] J.P. Lees, et al., BaBar Collaboration, Phys. Rev. D 86 (2012) 032013.

[11] VP correction values kindly provided by Fred Jegerlehner, http://wwwcom. physik.huberlin.de/ fjeger/alphaQEDn.uu.

[12] J.S. Schwinger,Particles, Sources and Fields, vol. 3, Addison-Wesley, Redwood City, USA, (1989), p. 99.

[13] M. Adinolfi, et al., KLOE Collaboration, Nucl. Instrum. Methods A 488 (2002) 51.

[14] M. Adinolfi, et al., KLOE Collaboration, Nucl. Instrum. Methods A 482 (2002) 364.

[15] F. Ambrosino, et al., KLOE Collaboration, Phys. Lett. B 670 (2009) 285.

[16] D.Babusci et al.,KLOE and KLOE-2 Collaborations, Physics Letters B 720 (2013) 336. 
[17] F. Ambrosino, et al., KLOE Collaboration, Phys. Lett. B 700 (2011) 102.

[18] R.R. Akhmetshin, et al., CMD-2 Collaboration, Phys. Lett. B 648 (2007) 28.

[19] R.R. Akhmetshin, et al., CMD-2 Collaboration, JETP Lett. 84 (2006) 413.

[20] M.N. Achasov, et al., SND Collaboration, J. Exp. Theor. Phys. 103 (2006) 380.

[21] B. Aubert, et al., BaBar Collaboration, Phys. Rev. Lett. 103 (2009) 231801.
[22] F. Ambrosino, et al., KLOE Collaboration, Eur. Phys. J. C 47 (2006) 589.

[23] B.E. Lautrup, E. de Rafael, Nuovo Cim. A 1 (1971) 238.

[24] S. Mueller, contribution to "Mini-Proceedings, 15th meeting of the Working Group on Rad. Corrections and MC Generators for Low Energies,", arXiv:1406.4639 [hep-ph]. Editors: S. E. Mueller and G. Venanzoni.

[25] A. Valassi NIM A 500 (2003) 391.

[26] G. D’Agostini NIM A 346 (1994) 306.

[27] G.J. Sakurai, Phys. Rev.Lett. 21 (1968) 244. 